\title{
Pour en savoir plus- Eléments bibliographiques sur le rôle des ONG dans la politique de développement
}

\section{OpenEdition}

1 Journals

Édition électronique

URL : http://journals.openedition.org/aspd/463

DOI : $10.4000 /$ aspd. 463

ISSN : 1663-9669

Éditeur

Institut de hautes études internationales et du développement

\section{Édition imprimée}

Date de publication : 1 novembre 2004

Pagination : 143-150

ISSN : 1660-5934

Référence électronique

«Pour en savoir plus- Eléments bibliographiques sur le rôle des ONG dans la politique de développement », Annuaire suisse de politique de développement [En ligne], 23-2 | 2004, mis en ligne le 10 mars 2010, consulté le 08 septembre 2020. URL : http://journals.openedition.org/aspd/463 ; DOI : https://doi.org/10.4000/aspd.463 


\section{Pour en savoir plus - Eléments bibliographiques sur le rôle des ONG dans la politique de développement}

La bibliographie se concentre sur les documents parus en Suisse, Allemagne, Autriche et France. Il s'agit d'une sélection qui n'est pas censée être exhaustive sur la question.

\section{Ouvrages}

Bundesministerium für wirtschaftliche Zusammenarbeit und Entwicklung, Medienhandbuch Entwicklungspolitik 2004-2005, Berlin, juin 2004, 520 p., téléchargeable sur Internet, <www.bmz.de>.

Annuaire sur la politique de coopération du Ministère allemand de la coopération au développement. Contient une partie sur le rôle des ONG de développement et une liste des principales adresses d'ONG allemandes. L'ouvrage analyse l'évolution des dons privés et les contributions gouvernementales aux ONG.

Centre tricontinental (ouvrage collectif), Les ONG: instruments du néo-libéralisme ou alternatives populaires ?, Paris, L'Harmattan, 1998, 194 p.

Commissariat général du Plan, L'Etat et les ONG : vers un partenariat efficace, Paris, La Documentation Française, 2002, 210 p. Rapport du groupe «pour un nouveau partenariat entre les organisations de solidarité internationale et les pouvoirs publics », groupe présidé par Jean-Claude Faure.

Coopération internationale pour la démocratie, Les ONG dans la restructuration des Nations unies, Coopération internationale pour la démocratie, $\mathrm{n}^{\circ} 5$ (Solagral, INRA, IATP, IBASE), septembre 1995, 256 p.

Deler, J.-P., Fauré, Y.-A., Piveteau A., Roca P.-J., ONG et développement. Société, économie, politique, coll. Hommes et Sociétés, Paris, Karthala, 1998, 684 p.

Edwards, Michael, NGO Rights and Responsibilities: A New Deal for Global Governance, London, Foreign Policy Center, 2000.

Edwards, Michael, Hulme, David, Non-Governmental Organisations - Performance and Accountability: Beyond the Magic Bullet, London, Earthscan Publications and Save the Children, 1998, 260 p.

Rare analyse de l'efficacité du travail des ONG.

Fisher, Julie, Non Governments: NGOs and the Political Development of the Third World, West Hartford, Connecticut, Kumarian Press, 1998, 234 p.

Analyse les contacts entre la politique et la croissance de la société civile, l'impact des ONG sur les gouvernements, et le rôle des ONG dans la démocratisation et le développement durable. 
Gerster, Richard, Nord-Süd-Politik: abschreiben oder investieren? Perspektiven der Schweizerischen Entwicklungszusammenarbeit, Zürich, Orell Füssli, 1995, $188 \mathrm{p}$.

Hanisch, Rolf, Wegner, Rodger (Hrsg.), Nichtregierungsorganisationen und Entwicklung. Auf dem Weg zu mehr Realismus, Schriften des Deutschen Übersee-Instituts, Nr. 28, Hamburg, Deutsches Übersee-Institut, 1994, 255 p.

Helmich, Henny, Bittner, Gerhard (Hrsg.), Der dritte Sektor in der Entwicklungspolitik. Zusammenarbeit zwischen Staat und Nicht-Regierungsorganisationen - NROs, Wien, Österreichische Forschungsstiftung für Entwicklungshilfe, 1996, $174 \mathrm{p}$.

Holenstein, René, Was kümmert uns die Dritte Welt. Zur Geschichte der internationalen Solidarität in der Schweiz, Zürich, Chronos Verlag, 1998, 264 p.

Historique du travail des mouvements de solidarité et des premières ONG suisses actives dans la coopération au développement et l'aide humanitaire.

Hudock, Ann C., NGOs and Civil Society: Democracy by Proxy, Cambridge; Oxford; Malden, Polity Press, 1999, 120 p.

Examine le rôle des ONG dans le renforcement de la société civile au Sud et le renforcement des capacités, ainsi que les relations entre ONG du Nord et du Sud.

Hug, Peter, Mesmer, Beatrix (Hrsg.), Von der Entwicklungshilfe zur Entwicklungspolitik, Studien und Quellen, Nr. 19, Bern, 1993.

Joly, Christian, Organisations non gouvernementales françaises et développement, Paris, Economica, 1985, 288 p.

Analyse du discours des ONG françaises de développement.

Klein, Ansgar, Der Diskurs der Zivilgesellschaft. Politische Hintergründe und demokratietheoretische Folgerungen, Opladen: Leske und Budrich-Verlag, 2001.

Landolf, Dani, Fankhauser, Michael, Gekaufte Entwicklungshilfe? Die (Un)Abhängigkeit der Nichtregierungsorganisationen von Geld und Einfluss der staatlichen Entwicklungspolitik, Köniz, Ed. Soziothek, Facharbeit Entwicklungsoziologie, 1996, $99 \mathrm{p}$.

Examen de la politique suisse de développement, de la place des œuvres d'entraide suisses dans la coopération et du financement des ONG.

Lecherey, Christian, Ryfman, Philippe, Action humanitaire et solidarité internationale: les ONG, Paris, Hatier, 1993, 80 p.

Le rôle des $0 N G$ dans l'aide humanitaire et dans la coopération.

Rossiaud, Jean, Mouvement social et mondialisation. Une illustration: le Helsinki Citizens Assembly, thèse de doctorat, $\mathrm{n}^{\circ}$ 421, Faculté des sciences économiques et sociales, Université de Genève, 1996. 
Rutz-Imhoof, Aschi, Tochtermann-Pedio, Daniel, Private und öffentliche Entwicklungshilfe der Schweiz. Helvetas und ihre Basis. Die Einbettung der Entwicklungshilfe in gesamtgesellschaftliche Zusammenhänge, Zürich, Eigenverlag, 1989, 381 p.

Ryfman, Philippe, Les ONG, coll. Repères, Paris, La Découverte, 2004, 124 p.

Origines et expansion des $\mathrm{ONG}$ au $x x^{e}$ siècle, réalité difficile à cerner et concept "ONG » difficile à définir. Examine trois domaines principaux: développement et humanitaire, environnement et droits humains; financement et concurrence entre ONG; rapports avec les organisations internationales.

Smillie, Ian, Helmich, Henny (eds.), Stakeholders: Government-NGO Partnerships for International Development, London, Earthscan Publications; OECD, 1999, $318 \mathrm{p}$.

Analyse les questions relatives aux relations entre gouvernements et $0 \mathrm{NG}$, avec des études de cas détaillées sur 22 pays (dont la Suisse), I'Union européenne et la Banque mondiale.

Wegner, Rodger, Nichtregierungsorganisationen und Entwicklungshilfe. Einführung und systematische Bibliographie, Schriften des Deutschen Übersee-Instituts, Nr. 20, Hamburg, Deutsches Übersee-Institut, 1993, 147 p.

Bibliographie commentée sur le rôle des ONG dans la coopération et études de cas par régions du monde.

- Nicht-Regierungsorganisationen als entwicklungspolitische Hoffnungsträger? Eine Studie zur Wirksamkeit privater Entwicklungshilfe auf den Philippinen, Demokratie und Entwicklung, Bd 10, Münster; Hamburg, Lit, 1993, 569 p.

World Bank, Working with NGOs : A Practical Guide to Operational Collaboration between the World Bank and Non-governmental Organizations, NGO Unit, Washington, D.C., World Bank, 1995.

\section{Documents, rapports}

Comeliau Christian, L'émergence internationale des organisations non gouvernementales, coll. Itinéraires notes et travaux, nº 68, Genève, iuéd, 2003, 25 p.

Commission Coopération-Développement, Argent et organisations de solidarité internationale 2000-2001, résultats de l'enquête de la Commission Coopération-Développement sur les ressources et dépenses des organisations de solidarité internationale en 2000 et 2001; mise en perspective des chiffres de 1991 à 2001, Paris, Commission Coopération-Développement, 2003, 32 p., téléchargeable sur Internet, <http://coordinationsud.datapps.com/data/ffiard/argent_et_ osi_2000-2001.pdf>.

Commission Coopération-Développement (Mission pour la coopération non gouvernementale, Ministère des affaires étrangères). Aide privée des ONG françaises.

DDC (Direction du développement et de la coopération), Aide de la Suisse aux pays en développement et aux pays en transition. Statistiques 2002, Berne, DDC, 2004, 200 p. 
iuéd (Institut universitaire d'études du développement), DDC, Aide suisse aux pays en développement et aux pays en transition 1998-2000, Genève, iuéd, 2002, $206 \mathrm{p}$.

Swissaid, Aide suisse aux pays en développement.

Les résultats des enquêtes annuelles menées en Suisse auprès des cantons/communes et auprès des ONG ont été publiés par Swissaid de 1961 à 1990, par l'iuéd de 1991 à 2000 et par la DDC depuis 2001. La brochure annuelle contient aussi des données sur l'aide multilatérale de la Suisse ainsi que des données détaillées sur l'aide bilatérale (pays par pays).

DDC (Direction du développement et de la coopération), Partenaires en Afrique. Quelle coopération pour quel développement?, série Ecrits sur le développement, $\mathrm{n}^{\circ}$ 5, Berne, DDC, 2002, $232 \mathrm{p}$.

Contient notamment un débat entre Olivier Berthoud (DDC) ("Six thèses sur l'avenir des ONG de développement et la coopération internationale») et Werner Külling (Helvetas) («Les ONG du Nord sauront s'adapter pour répondre à leurs partenaires »).

Fédération genevoise de coopération, Solidarité Genève Sud. Participation des collectivités publiques genevoises à la solidarité internationale en 2000, Genève, Fédération genevoise de coopération, 2001, 160 p.

Contient les adresses des 50 organisations membres de la FGC et la liste des projets soutenus par les collectivités publiques genevoises en l'an 2000. Adresses mises à jour sur <www.fgc.ch>.

Forum mondial de la société civile, Renforcer la coopération internationale. Rapport final, Genève, Mandat International, 2002.

Principaux résultats du Forum Mondial de la société civile qui s'est réuni à Genève en 2000.

Freymond, Jean F., Boyer, Brook, Les organisations non gouvernementales et la politique extérieure de la Suisse. Rapport de synthèse, Genève, CASIN (Centre d'études pratiques de la négociation internationale), 1998, 22 p.

Gerber, Maud, Le don aux ONG et les motivations des donateurs, mémoire de diplôme, Genève, iuéd, 2000, 44 p.

Helmich, Henny, Nouveaux partenariats pour la coopération au développement. Orientations des années 1980 et défis des années 1990, Centre de développement, Paris, OCDE, 1990, 39 p.

Lipietz, Alain, Pour le tiers secteur. L'économie sociale et solidaire, coll. Cahiers libres, Paris, La Documentation Française; La Découverte, 2001.

Ministère des affaires étrangères, Organisations de solidarité internationale et pouvoirs publics en Europe. Etude comparative sur les dispositifs d'appui et de concertation. Note de synthèse, Paris, Direction générale de la coopération internationale et du développement, 2001, 40 p.

Le rapport (en français et en anglais) établit une comparaison entre les pays européens du financement public des activités d'ONG (financement de l'Etat central).

Organisation des Nations unies, Rapport du Groupe de personnalités éminentes sur les relations entre l'Organisation des Nations unies et la société civile, $58^{\mathrm{e}}$ session de l'Assemblée générale de l'ONU, Renforcement du système des Nations unies, doc. A/58/817, <www.un.org/reform/panel.htm>. 
Petrella, Riccardo, Soulet, Marc-Henry, Longchamp, Albert, L'Etat démissionne? Place au privé! Les auvres d'entraide en question, Caritas, 1997, 56 p.

Face au démantèlement social, comment redéfinir le rôle des œuvres d'entraide (partenariats plus étroits avec les pouvoirs publics ou voie plus solitaire)? Doivent-elles prendre en charge une aide sociale que l’Etat délaisse?

Pfister, Dieter, Spendenmarkt Schweiz, 25 Jahre SMS: die Jubiläumsstudie, Basel, 1994.

Rosselet, Justine, ONG et management fondé sur la qualité: terre des hommes ou terre des normes?, coll. Itinéraires Etudes du développement, nº 19, Genève, iuéd, 2003, 76 p.

Fonctionnement des normes ISO, discours des ONG sur la certification et commentaire critique.

Woods, Adèle, Les ONG européennes actives dans le domaine du développement. Etat des lieux, Etudes du Centre de développement, Paris, OCDE, 2000, 96 p.

ZEWO (Bureau central des œuvres de bienfaisance), Institutions d'utilité publique en Suisse, Zurich, Orel Füssli Verlag; ZEWO, 1998, 288 p.

Un aperçu du travail d'utilité publique suisse accompagné de conseils aux donatrices et donateurs; liste d'adresses des ONG bénéficiant du label ZEWO.

\section{Répertoire d'adresses d'ONG et quelques portails Internet}

\section{Suisse}

Caritas, <www.caritas.ch>.

CASIN (Centre d'études pratiques de la négociation internationale), $<$ www.Casin.ch $>$.

Centre de documentation et d'analyse sur les ONG.

Chancellerie d'Etat, Répertoire de la Genève internationale 2004-2005, Genève, Chancellerie d'Etat; Club suisse de la presse, 2003.

Contient notamment une liste des organisations internationales gouvernementales, des organisations internationales non gouvernementales $(\mathrm{ONG})$, des agences spécialisées, des représentations permanentes de la Suisse à Genève, des Missions auprès de I'ONU et d'autres organisations internationales présentes à Genève.

CINFO (Centre d'information, de conseil et de formation - Professions de la coopération internationale), <www.cinfo.ch $>$.

Liens Internet sur des ONG et agences de coopération au développement de plusieurs pays européens.

Communauté de travail Swissaid, Action de carême, Pain pour le prochain, Helvetas, Caritas, EPER, <www.swisscoalition.ch>.

Déclaration de Berne, <www.laDB.ch $>$. 
Eco'Diagnostic, International Geneva Yearbook 2002-2003: Organization and Activities of International Institutions in Geneva, Geneva, United Nations; Eco' Diagnostic, 2002.

Répertoire d'adresses d'organisations internationales, d'institutions de recherche et d'organisations non gouvernementales à Genève.

iuéd (Institut universitaire d'études du développement), Répertoire des institutions Suisse - tiers-monde, sur le site Internet de l'iuéd <www.unige.ch/iued $>$.

Mandat International Genève, <www.mandatint.org $>$.

Centre d'accueil pour les délégations et organisations non gouvernementales. Cherche à faciliter la participation des délégués non gouvernementaux aux conférences internationales. L'une des bases de données les plus complètes sur les ONG suisses et internationales, classées par domaines d'activités et avec des liens Internet.

Menschenrechte Schweiz MERS, <www.humanrights.ch>.

Plate-forme des ONG suisses dans le domaine des droits de l'homme.

ZEWO (Bureau central des œuvres de bienfaisance), <www.zewo.ch>.

—, ZEWO-Handbuch «Gemeinnützige Institutionen in der Schweiz», Zürich, Orell Füssli Verlag; ZEWO, 2001.

Liste d'adresses des 300 organisations de bienfaisance bénéficiant du label du ZEWO.

\section{CAD/Europe}

CAD (Comité d'aide au développement), <www.oecd.org/dac>.

Centre de développement de l'OCDE, Directory of Development Organizations, $<$ www.euforic.org/detail_page.phtml?page=about_onesite $>$.

Répertoire par pays, mise à jour et en ligne sur Internet du Répertoire des organisations non gouvernementales actives dans le domaine du développement durable.

—, Répertoire des organisations non gouvernementales actives dans le domaine du développement durable, Paris, Centre de développement de l'OCDE, 1996.

-, Répertoire des organisations non gouvernementales de développement dans les pays membres de l'OCDE, Paris, Centre de développement de l'OCDE, 1990.

Euforic (Forum européen de coopération internationale), <www.euforic.org $>$.

The Reality of Aid, Independent Review of Poverty Reduction and development Assistance, <www.realityofaid.org $>$. 


\section{France}

Commission Coopération-Développement, Solidarité internationale. Répertoire des acteurs 2004, Paris, RITIMO, 2003, 236 p.

Annuaire détaillé des acteurs engagés dans la solidarité internationale, avec un index par pays et par secteur d'activité. Voir <www.ritimo.org>. Ce n'est pas un répertoire exhaustif (329 organisations dans l'édition 2004) car figurent les organisations qui ont répondu à un questionnaire.

Réseau d'associations de solidarité en France, <www.coordinationsud.org>. Coordination nationale des organisations françaises de solidarité internationale.

\section{Allemagne}

Bengo (Beratungsstelle für private Träger in der Entwicklungszusammenarbeit), $<$ www.paritaet.org/bengo $>$.

Bengo a été créé en 1988 pour conseiller les $0 N G$ et leur faciliter l'accès à des fonds publics.

DSE (Deutsche Stiftung für internationale Entwicklung), <www.dse.de/home. htm>.

Avec notamment une base de données sur les organisations de développement et sur les recherches en cours en Allemagne dans le domaine du développement et de l'aide au développement.

-, Institutionen der Entwicklungszusammenarbeit. Eine Auswahl, Bonn, Deutsche Stiftung für internationale Entwicklung, 8e édition, 2002, 364 p., numéro de commande DOK 281.

Verband Entwicklungspolitik Deutscher Nichtregierungsorganisationen, <www. venro.org >.

\section{Autriche}

ÖFSE (Österreichische Forschungsstiftung für Entwicklungshilfe), <www. oefse.at>.

ÖIS (Österreichisches Institut für Spendenwesen), <www.spendeninstitut.at>.

OneWorld Plattform Österreich, <www.oneworld.at>.

\section{Etats-Unis}

BBB Wise Giving Alliance, <www.give.org >.

Répertoire d'ONG américaines, avec notamment des rapports sur de nombreuses ONG.

NGOWatch, <www.ngowatch.org $>$.

Site Internet critique sur l'action des ONG. Un projet de I'«American Enterprise Institute for Public Research" et de la "Federalist Society for Law and Public Policy Studies".

Les articles publiés sur le site dénoncent par exemple la place trop importante prise par les ONG dans les organisations internationales et leur influence jugée excessive dans certains gouvernements. Certaines $0 N G$ assument des rôles quasi gouvernementaux. Quelle légitimité accorder aux ONG, qui dans 
beaucoup de pays ne sont soumises à aucun contrôle, ne doivent pas rendre de comptes et sont peu transparentes sur leurs modes de décision et leurs ressources financières?

\section{Nations unies}

NGLS (Service de liaison ONU-ONG), <www.un-ngls.org $>$.

Programme inter-agences des Nations unies pour faciliter le dialogue entre I'ONU et la communauté des ONG, avec des bureaux à Genève et New York. Publie notamment des newsletters (Go-Between et NGLS Roundup).

—, United Nations System: A Guide for NGOS, Geneva, NGLS, 2003.

UNO Panel of Eminent Persons on Civil Society and UN Relationships, $<$ www.un.org/reform/panel.htm>.

Réflexions du Groupe de personnalités éminentes sur les relations entre l'Organisation des Nations unies et la société civile. Avec le rapport final et les recommandations publiés en juin 2004. 